\title{
Extension, inflation and torsion of a residually-stressed circular cylindrical tube
}

\author{
${ }^{1}$ José Merodio and ${ }^{2}$ Ray W. Ogden \\ ${ }^{1}$ Department of Continuum Mechanics and Structures, E.T.S. Ingenieros Caminos, Canales y \\ Puertos, Universidad Politécnica de Madrid, 28040 Madrid, Spain \\ ${ }^{2}$ School of Mathematics and Statistics, University of Glasgow, Glasgow G12 8QW, UK \\ Email: raymond.ogden@glasgow.ac.uk
}

\begin{abstract}
In this paper we provide a new example of the solution of a finite deformation boundaryvalue problem for a residually-stressed elastic body. Specifically, we analyze the problem of the combined extension, inflation and torsion of a circular cylindrical tube subject to radial and circumferential residual stresses and governed by a residual-stress dependent nonlinear elastic constitutive law. The problem is first of all formulated for a general elastic strain-energy function, and compact expressions in the form of integrals are obtained for the pressure, axial load and torsional moment required to maintain the given deformation. For two specific simple prototype strain-energy functions that include residual stress the integrals are evaluated to give explicit closed-form expressions for the pressure, axial load and torsional moment. The dependence of these quantities on a measure of the radial strain is illustrated graphically for different values of the parameters (in dimensionless form) involved, in particular the tube thickness, the amount of torsion and the strength of the residual stress. The results for the two strain-energy functions are compared and also compared with results when there is no residual stress.
\end{abstract}

\section{Introduction}

The problem of extension and torsion of a solid cylinder for large deformations has been dealt with by many authors dating back to the theoretical and experimental work on rubber of Rivlin and colleagues $[1,2,3,4,5]$, with the material considered to be isotropic. See also the monograph by Green and Adkins [6], which provides several general formulas for this problem for both incompressible and compressible materials. An approach to the problem of extension and torsion of an incompressible isotropic cylinder based on principal axes was provided by Ogden and Chadwick [7].

For compressible isotropic elastic materials, apart from the early work described in [6], Horgan and Polignone [8] used the Blatz-Ko material model to examine loss of ellipticity. Kirkinis and Ogden [9] derived conditions on the form of strain-energy function for which the isochoric deformation of pure torsion superimposed on a uniform extension can be supported with vanishing traction on the lateral surfaces of the cylinder, and pure torsion of a compressible isotropic elastic material was also examined in [10].

A model of limiting chain extensibility for incompressible isotropic elastic materials was used by Horgan and Saccomandi in [11] in examining simple torsion, and by Kanner and Horgan in [12] for extension combined with torsion. Recently Horgan and Murphy [13, 14] considered the pure torsion and finite extension combined with torsion of incompressible solid cylinders reinforced with a single family of fibres arranged helically and locally in planes normal to the cylinder radius, with particular reference to axially aligned fibres.

For a circular cylindrical tube, as distinct from a solid cylinder, under extension, torsion and inflation, some general results for an incompressible isotropic elastic solid were provided by Rivlin [15]. A short contribution involving the combination of torsion and telescopic shear of a compressible isotropic tube of Blatz-Ko material was published by Zidi [16]. Some general results 
for transversely isotropic materials (both incompressible and compressible) with the direction of transverse isotropy aligned with the tube axis can be found in [6].

Two papers by Zidi $[17,18]$ examined the torsion of a compressible tube combined with either anti-plane shear or axial and azimuthal shear for a transversely isotropic material with the direction of transverse isotropy arranged helically normal to the radial direction, and in [19] the authors considered the combined torsion, azimuthal shear and radial expansion of an incompressible tube with the same arrangement of transverse isotropy. Recently El Hamdaoui et al. [20] analyzed the problem of extension and inflation of a tube for an incompressible transversely isotropic material and showed, in particular, that only certain directions of transverse isotropy are compatible with this deformation.

None of the aforementioned contributions took account of the possible existence of residual stresses. To motivate the need to include residual stresses, we mention first that for rubberlike materials used, for example, in bush mountings for the support of engines residual stresses are often introduced during the vulcanization process or in manufacturing (see, for example, [21, 22]). In this situation the residual stresses can have a detrimental influence on the material performance. Secondly, in soft biological tissues, residual stresses are produced during growth, development and remodelling and have an important positive influence on the mechanical behaviour of the tissues, as is well known for arteries, for example, and the heart. In each case the effect of residual stress on the material behaviour needs to be better understood.

In the context of artery walls, in particular, residual stresses are often accounted for by using a pre-deformation from a fictitious 'stress-free' configuration associated with the so-called opening angle method, which shows that when a ring of artery is cut radially it springs open, thereby releasing, at least in part, the residual stresses. The resulting opened sector is used to model the effect of residual stresses by deforming the sector into an intact ring (see, for example, [23, 24, 25], which were concerned with the extension and inflation of a tube). For the same deformation, Ogden and Schulze-Bauer [26] calculated the residual stress under the assumption that, at a typical physiological pressure, the circumferential stress in a single artery layer is uniform; see also [27]. A three-dimensional analysis of residual stress that takes account of the axial residual stress as well as the circumferential and radial stresses was reported in [28].

The opening angle method has also been employed in the series of papers [29, 30, 31, 32] in considering the torsion of a tube combined with a variety of shear-type deformations for compressible isotropic and transversely isotropic materials, again with the direction of transverse isotropy arranged helically normal to the radial direction.

However, to fully take account of residual stress a more general approach is necessary, and, in particular, the residual stress needs to feature in the constitutive law for the elastic response of the material, on which we focus in this paper, or for more general material response. Residual stress is regarded as a stress that is in equilibrium in the absence of external loads (tractions or body forces), as in the definition found in [33]. A formulation of the constitutive law for a residually stressed transversely isotropic hyperelastic material in terms of invariants has been provided by Hoger [34], and this formulation (and its specialization to the case without a preferred direction associated with transverse isotropy) has been used as a basis for analyzing various wave propagation problems in initially stressed (as distinct from residually stressed) elastic materials in $[35,36,37,38]$.

The general equilibrium equations satisfied by the residual stress in cylindrical polar coordinates and various special cases in which residual stress can in principle be determined from experimental set-ups have been provided by Hoger [39], while the problem in which residual stress is generated by eversion of a sphere has been examined in [40].

Recently, for a circular cylindrical tube, Merodio et al. [41] provided a general formulation of the elastic constitutive law for plane strain and solved the problem of the effect of residual stress on the azimuthal shear response of the tube. They also used the three-dimensional formulation to examine the problem of extension and torsion of a residually stressed solid cylinder. In [42] a solution of the problem of determining the effect of (a special choice of radial and circumferential) residual stress on the extension and inflation of a circular cylindrical tube with and without fibre reinforcement was derived. Otherwise, as far as we are aware, there are no papers in the literature 
that solve specific finite deformation elastic boundary-value problems where the residual stress is explicitly included in the constitutive law.

The purpose of the present work is to extend this short catalogue of solutions by incorporating residual stresses into the analysis of the problem of extension, inflation and torsion of a circular cylindrical tube under finite deformations.

The structure of the paper is as follows. In Sect. 2 the geometry and kinematics of the problem are summarized, specifically the geometry of a circular cylindrical tube which retains its circular cylindrical shape under extension, inflation and torsion. In Sect. 3 residual stress is introduced along with the equilibrium equations, and a particular form of residual stress appropriate for the considered geometry is discussed. The effect of residual stresses on the constitutive law has a parallel with the effect of fibre reinforcement in that the residual stress tensor can be regarded as a generalized structure tensor from which certain invariants can be formed. The independent invariants associated with the combination of deformation and residual stress are listed in Sect. 4 for an incompressible material, on which we focus here. The form of the strain-energy function in terms of invariants is given, along with the general expression for Cauchy stress. In the reference configuration the latter reduces to the residual stress and thereby puts restrictions on the form of the energy function and its derivatives with respect to the invariants in the reference configuration.

The theory of Sect. 4 is applied in Sect. 5 to the geometry and deformation considered herein. General formulas, in the form of integrals, for the pressure $P$, the axial load $N$, and the torsional moment $M$ required to maintain the considered deformation are given in a simple form for a general form of strain-energy function that is expressed in terms of three key kinematic variables.

In Sect. 6 two prototype forms of strain-energy function that include dependence on the residual stress in a very simple way are introduced, and these enable explicit formulas for $P, N, M$ to be obtained by evaluating the integrals in closed form. These formulas are used to obtain numerical results to illustrate the effect on $P, N, M$ of the residual stress compared with results in its absence for different choices of the geometric parameters and the magnitude of the residual stress.

Finally, in Sect. 7, some concluding remarks are provided and we also consider briefly the strong ellipticity character of the constitutive laws.

\section{Kinematics and geometry}

For full details of the kinematics involved in finite deformation theory we refer to the standard text [43]. Here we just summarize the kinematics that are needed for the problem considered herein.

Consider a material continuum which, when unstressed and unstrained, occupies the reference configuration $\mathcal{B}_{\mathrm{r}}$. Let a typical material point in this configuration be identified by its position vector $\mathbf{X}$. The corresponding position vector in the deformed configuration $\mathcal{B}$ is denoted $\mathbf{x}$ and the deformation from $\mathcal{B}_{\mathrm{r}}$ to $\mathcal{B}$ is written $\mathbf{x}=\chi(\mathbf{X})$, where the vector function $\chi$ is referred to as the deformation (attention is confined to quasi-static deformations here). The deformation gradient tensor, denoted $\mathbf{F}$, is given by

$$
\mathbf{F}=\operatorname{Grad} \chi(\mathbf{X}),
$$

where Grad is the gradient operator with respect to $\mathbf{X}$. The associated right and left CauchyGreen deformation tensors, denoted $\mathbf{C}$ and $\mathbf{B}$ respectively, are defined by

$$
\mathbf{C}=\mathbf{F}^{\mathrm{T}} \mathbf{F}=\mathbf{U}^{2}, \quad \mathbf{B}=\mathbf{F F}^{\mathrm{T}}=\mathbf{V}^{2},
$$

where ${ }^{\mathrm{T}}$ signifies the transpose of a second-order tensor, $\mathbf{U}$ and $\mathbf{V}$, respectively, are the right and the left stretch tensors, which are positive definite and symmetric and come from the polar decompositions $\mathbf{F}=\mathbf{R} \mathbf{U}=\mathbf{V R}, \mathbf{R}$ being a proper orthogonal tensor. For a homogeneous incompressible nonlinearly isotropic elastic solid, the elastic stored energy (defined per unit volume) depends on only two invariants, which are the principal invariants of $\mathbf{C}$ (equivalently of $\mathbf{B}$ ), defined by

$$
I_{1}=\operatorname{tr}(\mathbf{C})=\lambda_{1}^{2}+\lambda_{2}^{2}+\lambda_{3}^{2}, \quad I_{2}=\frac{1}{2}\left[(\operatorname{tr} \mathbf{C})^{2}-\operatorname{tr}\left(\mathbf{C}^{2}\right)\right]=\lambda_{1}^{2} \lambda_{2}^{2}+\lambda_{1}^{2} \lambda_{3}^{2}+\lambda_{2}^{2} \lambda_{3}^{2},
$$


where $\lambda_{i}>0, i \in\{1,2,3\}$, are the principal stretches, i.e. the eigenvalues of $\mathbf{U}$ and $\mathbf{V}$. The incompressibility constraint may be written as

$$
\operatorname{det} \mathbf{F}=1, \quad \text { or } \quad \lambda_{1} \lambda_{2} \lambda_{3}=1,
$$

equivalently in terms of $\mathbf{F}$ and the principal stretches, respectively.

\subsection{Combined extension, inflation and torsion}

We now consider a circular cylindrical tube, which, in terms of cylindrical polar coordinates $(R, \Theta, Z)$, is defined by

$$
A \leq R \leq B, \quad 0 \leq \Theta \leq 2 \pi, \quad 0 \leq Z \leq L
$$

in the reference configuration $\mathcal{B}_{\mathrm{r}}$, where $A$ and $B$ are the internal and external radii and $L$ is the length of the tube. The position vector $\mathbf{X}$ of a point of the tube is given by

$$
\mathbf{X}=R \mathbf{E}_{R}+Z \mathbf{E}_{Z}
$$

where $\mathbf{E}_{R}$ and $\mathbf{E}_{Z}$ are the unit basis vectors associated with $R$ and $Z$, respectively. We also denote by $\mathbf{E}_{\Theta}$ the corresponding unit vector associated with $\Theta$.

The position vector $\mathbf{x}$ in the deformed tube is written

$$
\mathbf{x}=r \mathbf{e}_{r}+z \mathbf{e}_{z},
$$

where we make use of cylindrical polar coordinates $(r, \theta, z)$ in $\mathcal{B}$, which are associated with unit basis vectors $\mathbf{e}_{r}, \mathbf{e}_{\theta}, \mathbf{e}_{z}$, respectively. The (isochoric) deformation consisting of axial extension, radial inflation and a superimposed torsion is defined by

$$
r=\sqrt{a^{2}+\lambda_{z}^{-1}\left(R^{2}-A^{2}\right)}, \quad \theta=\Theta+\psi \lambda_{z} Z, \quad z=\lambda_{z} Z,
$$

where $\lambda_{z}$ is the (uniform) axial stretch of the cylinder, $\psi$ is the torsional deformation per unit deformed length. Plane cross sections of the tube remain plane, and an initial radius at axial coordinate $Z$ turns through an angle $\psi z$ after axial extension. The deformed geometry of the tube is defined by

$$
a \leq r \leq b, \quad 0 \leq \theta \leq 2 \pi, \quad 0 \leq z \leq l=\lambda_{z} L .
$$

For this deformation the deformation gradient is calculated explicitly as

$$
\mathbf{F}=\lambda_{r} \mathbf{e}_{r} \otimes \mathbf{E}_{R}+\lambda_{\theta} \mathbf{e}_{\theta} \otimes \mathbf{E}_{\Theta}+\lambda_{z} \mathbf{e}_{z} \otimes \mathbf{E}_{Z}+\lambda_{z} \gamma \mathbf{e}_{\theta} \otimes \mathbf{E}_{Z},
$$

where we have defined $\gamma$ as $\gamma=\psi r$ and $\lambda_{r}, \lambda_{\theta}$ and $\lambda_{z}$ are the principal stretches in the radial, azimuthal and axial directions prior to application of the torsion. In particular, $\lambda_{\theta}=r / R$. Once the torsion is applied $\lambda_{\theta}$ and $\lambda_{z}$ are no longer principal stretches. Nevertheless, the incompressibility constraint $(4)_{2}$ becomes

$$
\lambda_{r} \lambda_{\theta} \lambda_{z}=1
$$

which is independent of $\gamma$. In general, application of the torsion will change the geometry given by (9), but here we fix the length of the tube during torsion at zero pressure and the internal radius is adjusted accordingly prior to application of a pressure to ensure that the circular cylindrical configuration is maintained with appropriate axial load and torsional moment. The deformation tensors (2) are calculated as

$$
\begin{aligned}
\mathbf{C} & =\lambda_{r}^{2} \mathbf{E}_{R} \otimes \mathbf{E}_{R}+\lambda_{\theta}^{2} \mathbf{E}_{\Theta} \otimes \mathbf{E}_{\Theta}+\lambda_{z}^{2}\left(1+\gamma^{2}\right) \mathbf{E}_{Z} \otimes \mathbf{E}_{Z} \\
& +\gamma \lambda_{z} \lambda_{\theta}\left(\mathbf{E}_{\Theta} \otimes \mathbf{E}_{Z}+\mathbf{E}_{Z} \otimes \mathbf{E}_{\Theta}\right) \\
\mathbf{B} & =\lambda_{r}^{2} \mathbf{e}_{r} \otimes \mathbf{e}_{r}+\left(\lambda_{\theta}^{2}+\gamma^{2} \lambda_{z}^{2}\right) \mathbf{e}_{\theta} \otimes \mathbf{e}_{\theta}+\lambda_{z}^{2} \mathbf{e}_{z} \otimes \mathbf{e}_{z} \\
& +\gamma \lambda_{z}^{2}\left(\mathbf{e}_{\theta} \otimes \mathbf{e}_{z}+\mathbf{e}_{z} \otimes \mathbf{e}_{\theta}\right) .
\end{aligned}
$$

By referring to the expression for $\mathbf{C}$ above, the definition $(3)_{1},(4)_{2}$ and (11) it may be deduced that the principal stretches $\lambda_{1}, \lambda_{2}, \lambda_{3}$, with a suitable ordering, can be taken to satisfy

$$
\lambda_{1}=\lambda_{r}, \quad \lambda_{2} \lambda_{3}=\lambda_{\theta} \lambda_{z}, \quad \lambda_{2}^{2}+\lambda_{3}^{2}=\lambda_{\theta}^{2}+\lambda_{z}^{2}\left(\gamma^{2}+1\right) .
$$




\section{Equilibrium and residual stress}

Throughout this paper we assume that there are no body forces present. The Cauchy stress $\boldsymbol{\sigma}$ and the nominal stress $\mathbf{S}$ then satisfy the equilibrium equations

$$
\operatorname{div} \boldsymbol{\sigma}=\mathbf{0}, \quad \operatorname{Div} \mathbf{S}=\mathbf{0},
$$

respectively, where div and Div are the divergence operators with respect to $\mathbf{x} \in \mathcal{B}$ and $\mathbf{X} \in \mathcal{B}_{\mathrm{r}}$, respectively, and are connected by $\boldsymbol{\sigma}=\mathbf{F S}$ for the considered incompressible material. In the absence of intrinsic couple stresses, $\boldsymbol{\sigma}$ is symmetric and hence $(\mathbf{F S})^{\mathrm{T}}=\mathbf{F S}$. Appropriate traction boundary conditions should be imposed on all or part of the boundary $\partial \mathcal{B}$ of $\mathcal{B}$, equivalently on the boundary $\partial \mathcal{B}_{\mathrm{r}}$ of $\mathcal{B}_{\mathrm{r}}$, but we do not specify these at this point. We refer to [43] for possible options for general problems.

We now assume that the reference configuration $\mathcal{B}_{\mathrm{r}}$ is residually stressed, with the residual stress tensor denoted by $\boldsymbol{\tau}$. In this configuration $\mathbf{S}=\boldsymbol{\sigma}=\boldsymbol{\tau}$, i.e. there is no distinction between different measures of stress since the deformation is measured from $\mathcal{B}_{\mathrm{r}}$.

The source of $\boldsymbol{\tau}$ does not concern us here. It may be associated with some prior material processing, plastic deformation or manufacturing process, for example, and is assumed to be known. It arises in the absence of body forces and surface tractions on the boundary $\partial \mathcal{B}_{\mathrm{r}}$ of the material body $\mathcal{B}_{\mathrm{r}}$. It is also assumed that it is not accompanied by intrinsic couple stresses, so that it is symmetric $\left(\boldsymbol{\tau}^{\mathrm{T}}=\boldsymbol{\tau}\right)$ and therefore the rotational balance equations are satisfied in $\mathcal{B}_{\mathrm{r}}$ as well as the equilibrium equation

$$
\operatorname{Div} \boldsymbol{\tau}=\mathbf{0}
$$

Since there are no surface tractions, then, by definition, $\tau$ must also satisfy the boundary condition

$$
\tau \mathbf{N}=\mathbf{0} \quad \text { on } \quad \partial \mathcal{B}_{\mathrm{r}}
$$

Note that $\boldsymbol{\tau}$ is a residual stress in the sense of Hoger [33] and is distinguished from other types of initial stress, which may be associated with surface tractions. We emphasize at this point that residual stresses are necessarily non-uniform and geometry dependent, and the elastic response of a residually-stressed material body is therefore inhomogeneous.

For the considered circular cylindrical geometry it is appropriate to assume that the only components of residual stress are $\tau_{R R}, \tau_{\Theta \Theta}$ and $\tau_{Z Z}$, i.e. there is no residual shear stress, an assumption that is compatible with the boundary condition (16) that the residual stress must satisfy. However, the $Z$ component of the equilibrium equation and corresponding boundary conditions $\tau_{Z Z}=0$ on the ends of the (finite length) tube then ensure that $\tau_{Z Z} \equiv 0$. The remaining components, $\tau_{R R}$ and $\tau_{\Theta \Theta}$, can then be taken to depend only on $R$, and the non-trivial component of the equilibrium equation (15) is the radial equation

$$
\frac{\mathrm{d} \tau_{R R}}{\mathrm{~d} R}+\frac{1}{R}\left(\tau_{R R}-\tau_{\Theta \Theta}\right)=0,
$$

with accompanying boundary conditions from (16):

$$
\tau_{R R}=0 \quad \text { on } R=A, B .
$$

Note that if $\tau_{R R}$ is known then $\tau_{\Theta \Theta}$ is determined by $(17)$ as $\mathrm{d}\left(R \tau_{R R}\right) / \mathrm{d} R$.

\section{Constitutive laws}

For a homogeneous incompressible elastic solid the strain energy is a function only of the deformation gradient $\mathbf{F}$, and we write the strain-energy function as $W(\mathbf{F})$ per unit volume, although, by objectivity, $W$ depends on $\mathbf{F}$ only through the right Cauchy-Green tensor $\mathbf{C}$ defined in (2). The Cauchy and nominal stress tensors $\boldsymbol{\sigma}$ and $\mathbf{S}$ are given by

$$
\boldsymbol{\sigma}=\mathbf{F} \frac{\partial W}{\partial \mathbf{F}}-p \mathbf{I}, \quad \mathbf{S}=\frac{\partial W}{\partial \mathbf{F}}-p \mathbf{F}^{-1},
$$


where $p$ is a Lagrange multiplier associated with the incompressibility constraint $(4)_{1}$ and $\mathbf{I}$ is the identity tensor in $\mathcal{B}$.

When the material is residually stressed, then it is also inhomogeneous. We consider its response still to be elastic relative to $\mathcal{B}_{\mathrm{r}}$, with dependence on $\mathbf{X}$ through $\boldsymbol{\tau}(\mathbf{X})$, and we account for this by including $\boldsymbol{\tau}$ in the argument of $W$. Thus, we write

$$
W=W(\mathbf{F}, \boldsymbol{\tau}) .
$$

Note that this is automatically objective since $\boldsymbol{\tau}$ is unaffected by rotations in the deformed configuration $\mathcal{B}$ and $W$ depends on $\mathbf{F}$ only via $\mathbf{C}=\mathbf{F}^{\mathrm{T}} \mathbf{F}$.

We also note that if the material has no intrinsic preferred directions in $\mathcal{B}_{\mathrm{r}}$ other than those associated with $\boldsymbol{\tau}$ (its eigenvectors) then the elastic properties of the material relative to $\mathcal{B}_{\mathrm{r}}$ are anisotropic, i.e. $\boldsymbol{\tau}$ has an effect on the constitutive law analogous to that of a structure tensor associated with preferred directions. We shall elaborate on this point shortly.

The presence of $\boldsymbol{\tau}$ does not affect the formula $(19)_{1}$ for the Cauchy stresses except by the dependence of $W$ on $\boldsymbol{\tau}$. It given by

$$
\boldsymbol{\sigma}=\mathbf{F} \frac{\partial W}{\partial \mathbf{F}}(\mathbf{F}, \boldsymbol{\tau})-p \mathbf{I} .
$$

When $\mathbf{F}=\mathbf{I}$ this reduces to

$$
\boldsymbol{\tau}=\frac{\partial W}{\partial \mathbf{F}}(\mathbf{I}, \boldsymbol{\tau})-p^{(\mathrm{r})} \mathbf{I},
$$

where $p^{(\mathrm{r})}$ is the value of $p$ in $\mathcal{B}_{\mathrm{r}}$. Equation (22) imposes restrictions on the combination of $W$ and $\boldsymbol{\tau}$, restrictions that will be made more explicit in the following subsection.

Any symmetric second-order tensor can be expressed in spectral form in terms of its eigenvalues and eigenvectors. In particular, in terms of its eigenvalues, $\tau_{1}, \tau_{2}, \tau_{3}$ and (unit) eigenvectors $\mathbf{M}_{1}, \mathbf{M}_{2}, \mathbf{M}_{3}$, say, $\boldsymbol{\tau}$ can be written

$$
\boldsymbol{\tau}=\tau_{1} \mathbf{M}_{1} \otimes \mathbf{M}_{1}+\tau_{2} \mathbf{M}_{2} \otimes \mathbf{M}_{2}+\tau_{3} \mathbf{M}_{3} \otimes \mathbf{M}_{3}
$$

and when $\boldsymbol{\tau}$ is included in $W$, each $\mathbf{M}_{i} \otimes \mathbf{M}_{i}, i=1,2,3$, has a role similar to a structure tensor associated with a single preferred direction, as for a fibre-reinforced material (see, for example, [44]). However, the $\mathbf{M}_{i} \otimes \mathbf{M}_{i}$ are not all independent since they satisfy the identity

$$
\mathbf{M}_{1} \otimes \mathbf{M}_{1}+\mathbf{M}_{2} \otimes \mathbf{M}_{2}+\mathbf{M}_{3} \otimes \mathbf{M}_{3}=\mathbf{I},
$$

where $\mathbf{I}$ is the identity tensor in $\mathcal{B}_{\mathrm{r}}$. Thus, $\boldsymbol{\tau}$ generates invariants, which contribute to the independent variables in the functional dependence of $W$. In particular, by specializing $\tau$ to a rank-one tensor, say $\mathbf{M} \otimes \mathbf{M}$, we recover the invariants associated with transverse isotropy. We now consider an invariant formulation for $W$ with the invariants generated by $\mathbf{C}$ and $\boldsymbol{\tau}$.

\subsection{Invariant Formulation}

With $W$ written explicitly as $W(\mathbf{C}, \boldsymbol{\tau})$, it is clear that $W$ is automatically objective. In the absence of any intrinsic material symmetry $W$ is an isotropic function of the combination of the $\mathbf{C}$ and $\boldsymbol{\tau}$ according to the theory of Spencer [45] and must therefore satisfy the symmetry condition

$$
W\left(\mathbf{Q}^{*} \mathbf{C Q}^{* \mathrm{~T}}, \mathbf{Q}^{*} \boldsymbol{\tau} \mathbf{Q}^{* \mathrm{~T}}\right)=W(\mathbf{C}, \boldsymbol{\tau}),
$$

for all orthogonal $\mathbf{Q}^{*}$ in $\mathcal{B}_{\mathrm{r}}$. For an incompressible material this is equivalent to the dependence of $W$ on nine invariants of $\mathbf{C}, \boldsymbol{\tau}$ and their combination. These are typically taken to be, for $\mathbf{C}$,

$$
I_{1}=\operatorname{tr} \mathbf{C}, \quad I_{2}=\frac{1}{2}\left[(\operatorname{tr} \mathbf{C})^{2}-\operatorname{tr}\left(\mathbf{C}^{2}\right)\right],
$$


as for an isotropic material (note that the third invariant $I_{3}=\operatorname{det} \mathbf{C}$ is equal to 1 for an incompressible material). Similarly for $\boldsymbol{\tau}$ (except that there is no counterpart for $\boldsymbol{\tau}$ of the incompressibility constraint $\operatorname{det} \mathbf{C}=1$ ),

$$
I_{4}=\left\{I_{41}, I_{42}, I_{43}\right\} \equiv\left\{\operatorname{tr} \boldsymbol{\tau}, \frac{1}{2}\left[(\operatorname{tr} \boldsymbol{\tau})^{2}-\operatorname{tr}\left(\boldsymbol{\tau}^{2}\right)\right], \operatorname{det} \boldsymbol{\tau}\right\}
$$

which are collectively denoted $I_{4}$. Then, we take the set of independent invariants involving the combination of $\mathbf{C}$ and $\boldsymbol{\tau}$ to be

$$
I_{5}=\operatorname{tr}(\boldsymbol{\tau} \mathbf{C}), \quad I_{6}=\operatorname{tr}\left(\boldsymbol{\tau} \mathbf{C}^{2}\right), \quad I_{7}=\operatorname{tr}\left(\boldsymbol{\tau}^{2} \mathbf{C}\right), \quad I_{8}=\operatorname{tr}\left(\boldsymbol{\tau}^{2} \mathbf{C}^{2}\right) .
$$

Here we have considered a specialization of the list of invariants given by Hoger [34] for a transversely isotropic material with residual stress, and also used in [46].

We emphasize that the above set of nine invariants, or an equivalent set of alternative invariants, forms a complete set of invariants of $\mathbf{C}$ and $\boldsymbol{\tau}$ in three dimensions. When the dimension of the considered problem is reduced from three to two, such as for plane strain, the number of independent invariants is reduced, as detailed in [41].

We may now regard $W$ as a function of the above invariants. Thus, we take $W=W\left(I_{1}, I_{2}, I_{4}, I_{5}, I_{6}, I_{7}, I_{8}\right)$, and in the following we use the notation $W_{i}=\partial W / \partial I_{i}, i=1,2,5,6,7,8$. On evaluation of $\partial I_{i} / \partial \mathbf{F}, i=1,2,5,6,7,8$, the Cauchy stress tensor (21) then expands out as

$$
\begin{aligned}
\boldsymbol{\sigma} & =2 W_{1} \mathbf{B}+2 W_{2}\left(I_{1} \mathbf{B}-\mathbf{B}^{2}\right)+2 W_{5} \boldsymbol{\Sigma}+2 W_{6}(\boldsymbol{\Sigma} \mathbf{B}+\mathbf{B} \boldsymbol{\Sigma}) \\
& +2 W_{7} \boldsymbol{\Sigma} \mathbf{B}^{-1} \boldsymbol{\Sigma}+2 W_{8}\left(\boldsymbol{\Sigma} \mathbf{B}^{-1} \boldsymbol{\Sigma} \mathbf{B}+\mathbf{B} \boldsymbol{\Sigma} \mathbf{B}^{-1} \boldsymbol{\Sigma}\right)-p \mathbf{I},
\end{aligned}
$$

in which we have introduced the notation $\boldsymbol{\Sigma}=\mathbf{F} \boldsymbol{\tau} \mathbf{F}^{\mathrm{T}}$ for the Eulerian tensor which is the push forward of $\boldsymbol{\tau}$ from $\mathcal{B}_{\text {r }}$ to $\mathcal{B}$. We also recall that $\mathbf{B}=\mathbf{F F}^{\mathrm{T}}$ is the left Cauchy-Green tensor.

The invariants of $\boldsymbol{\tau}$ are not affected by the deformation, while in the configuration $\mathcal{B}_{\mathrm{r}}$ the other invariants reduce to

$$
I_{1}=I_{2}=3, \quad I_{5}=I_{6}=\operatorname{tr} \boldsymbol{\tau}, \quad I_{7}=I_{8}=\operatorname{tr}\left(\boldsymbol{\tau}^{2}\right) .
$$

By evaluating (29) in $\mathcal{B}_{\mathrm{r}}$ we obtain the specialization of $(22)$ in the form

$$
\boldsymbol{\tau}=2\left(W_{1}+2 W_{2}-p^{(\mathrm{r})} \mathbf{I}+2\left(W_{5}+2 W_{6}\right) \boldsymbol{\tau}+2\left(W_{7}+2 W_{8}\right) \boldsymbol{\tau}^{2},\right.
$$

where all $W_{i}, i \in\{1,2,5,6,7,8\}$, are evaluated for the invariants given by (30). Thus, following [35], we obtain the residual stress dependent restrictions

$$
2 W_{1}+4 W_{2}-p^{(\mathrm{r})}=0, \quad 2\left(W_{5}+2 W_{6}\right)=1, \quad W_{7}+2 W_{8}=0,
$$

on the strain-energy function in $\mathcal{B}_{\mathrm{r}}$, where again the $W_{i}$ are evaluated in $\mathcal{B}_{\mathrm{r}}$.

\section{Application to combined extension, inflation and torsion}

For the considered deformation, with $\mathbf{C}$ given by $(12)_{1}$ and residual stress components $\tau_{R R}$ and $\tau_{\Theta \Theta}$, the invariants are given by

$$
\begin{aligned}
I_{1} & =\lambda_{r}^{2}+\lambda_{\theta}^{2}+\lambda_{z}^{2}\left(1+\gamma^{2}\right), \quad I_{2}=\lambda_{\theta}^{2} \lambda_{z}^{2}+\lambda_{r}^{2} \lambda_{z}^{2}\left(1+\gamma^{2}\right)+\lambda_{r}^{2} \lambda_{\theta}^{2}, \\
I_{41} & =\tau_{R R}+\tau_{\Theta \Theta}, \quad I_{42}=\tau_{R R} \tau_{\Theta \Theta}, \quad I_{43}=0 \\
I_{5} & =\lambda_{r}^{2} \tau_{R R}+\lambda_{\theta}^{2} \tau_{\Theta \Theta}, \quad I_{6}=\lambda_{r}^{4} \tau_{R R}+\lambda_{\theta}^{2}\left(\lambda_{\theta}^{2}+\gamma^{2} \lambda_{z}^{2}\right) \tau_{\Theta \Theta} \\
I_{7} & =\lambda_{r}^{2} \tau_{R R}^{2}+\lambda_{\theta}^{2} \tau_{\Theta \Theta}^{2}, \quad I_{8}=\lambda_{r}^{4} \tau_{R R}^{2}+\lambda_{\theta}^{2}\left(\lambda_{\theta}^{2}+\gamma^{2} \lambda_{z}^{2}\right) \tau_{\Theta \Theta}^{2} .
\end{aligned}
$$

They depend on just three independent deformation variables, which we take as $\lambda_{\theta}, \lambda_{z}$ and $\gamma$, together with $\tau_{R R}$ and $\tau_{\Theta \Theta}, \lambda_{r}$ being given by the incompressibility condition (11) in terms of 
$\lambda_{\theta}$ and $\lambda_{z}$. We therefore write the strain energy as a function of these variables, specifically $\hat{W}\left(\lambda_{\theta}, \lambda_{z}, \gamma, \tau_{R R}, \tau_{\Theta \Theta}\right)$, which is defined by

$$
\hat{W}\left(\lambda_{\theta}, \lambda_{z}, \gamma, \tau_{R R}, \tau_{\Theta \Theta}\right)=W\left(I_{1}, I_{2}, I_{4}, I_{5}, I_{6}, I_{7}, I_{8}\right),
$$

with $I_{1}, I_{2}, I_{4}, I_{5}, I_{6}, I_{7}, I_{8}$ given by (33).

Then, a straightforward calculation using the appropriate specialization of the components of the Cauchy stress in (29), the expression for $\mathbf{B}$ in (12) and the component matrix of $\boldsymbol{\Sigma}=\mathbf{F} \boldsymbol{\tau} \mathbf{F}^{\mathrm{T}}$, which has the diagonal form $\operatorname{diag}\left[\lambda_{r}^{2} \tau_{R R}, \lambda_{\theta}^{2} \tau_{\Theta \Theta}, 0\right]$, leads to the compact formulas

$$
\begin{aligned}
& \sigma_{\theta \theta}-\sigma_{r r}=\lambda_{\theta} \frac{\partial \hat{W}}{\partial \lambda_{\theta}}+\gamma \frac{\partial \hat{W}}{\partial \gamma}, \quad \sigma_{\theta z}=\frac{\partial \hat{W}}{\partial \gamma}, \\
& \sigma_{z z}-\sigma_{r r}=\lambda_{z} \frac{\partial \hat{W}}{\partial \lambda_{z}}-\gamma \frac{\partial \hat{W}}{\partial \gamma},
\end{aligned}
$$

with $\sigma_{r \theta}=0$ and $\sigma_{r z}=0$. These formulas are the same as those for a fibre-reinforced material with a single family of fibres except that in that case $\sigma_{r \theta}$ and $\sigma_{r z}$ are in general non-zero [20], although the content is different since $\hat{W}$ is different - it involves residual stress instead of the transverse isotropy associated with a single preferred direction. By default these formulas also apply in the isotropic case, which is recovered by setting the residual stress to zero.

\subsection{Equilibrium and boundary loads}

Since $\lambda_{z}$ is a constant and $\lambda_{\theta}$ and $\gamma$ depend only on $r$ (equivalently $R$ ) while $\sigma_{r \theta}=\sigma_{r z}=0$ the equilibrium equation $(14)_{1}$ reduces to just one scalar equation, namely

$$
r \frac{\mathrm{d}}{\mathrm{d} r}\left(\sigma_{r r}\right)+\sigma_{r r}-\sigma_{\theta \theta}=0,
$$

which can be integrated to give

$$
\sigma_{r r}-\sigma_{r r}(a)=\int_{a}^{r}\left(\sigma_{\theta \theta}-\sigma_{r r}\right) \frac{\mathrm{d} r}{r},
$$

where $\sigma_{r r}(a)$ is the value of the radial stress $\sigma_{r r}$ on the boundary $r=a$.

We now consider the situation in which the inner surface $r=a$ is subject to a pressure $P$ and no traction is applied on $r=b$. Then $\sigma_{r r}(a)=-P$ and $\sigma_{r r}=0$ on $r=b$, and, with the help of $(35)_{1}$, Eq. (37) becomes

$$
P=\int_{a}^{b}\left(\lambda_{\theta} \frac{\partial \hat{W}}{\partial \lambda_{\theta}}+\gamma \frac{\partial \hat{W}}{\partial \gamma}\right) \frac{\mathrm{d} r}{r}
$$

The resultant moment $M$ on any cross section of the tube is given by

$$
M=\int_{a}^{b} \int_{0}^{2 \pi} \sigma_{\theta z} r^{2} \mathrm{~d} r \mathrm{~d} \theta=2 \pi \int_{a}^{b} \frac{\partial \hat{W}}{\partial \gamma} r^{2} \mathrm{~d} r
$$

where the expression $(35)_{2}$ has been used.

The resultant axial load $N$ on any cross section is given by

$$
N=\int_{a}^{b} \int_{0}^{2 \pi} \sigma_{z z} r \mathrm{~d} r \mathrm{~d} \theta=2 \pi \int_{a}^{b} \sigma_{z z} r \mathrm{~d} r
$$

On use of $(36)_{1}$, the boundary values of $\sigma_{r r}$, and (35), this leads to an expression for the so-called reduced axial load $F$, which is defined as the the total axial load $N$ on the ends of a tube with closed ends reduced by the contribution of the pressure $P$. This is given by

$$
F \equiv N-\pi a^{2} P=\pi \int_{a}^{b}\left(2 \lambda_{z} \frac{\partial \hat{W}}{\partial \lambda_{z}}-\lambda_{\theta} \frac{\partial \hat{W}}{\partial \lambda_{\theta}}-3 \gamma \frac{\partial \hat{W}}{\partial \gamma}\right) r \mathrm{~d} r
$$


The above formulas for $P, M$ and $F$ are the same formulas as for an isotropic material or for a transversely isotropic material [20], but, as for (35), the content is different.

We also note that, since $\gamma=\psi r$, then, by Eq. (39), (41) can be written as

$$
F=\pi \int_{a}^{b}\left(2 \lambda_{z} \frac{\partial \hat{W}}{\partial \lambda_{z}}-\lambda_{\theta} \frac{\partial \hat{W}}{\partial \lambda_{\theta}}\right) r \mathrm{~d} r-\frac{3}{2} \psi M .
$$

\section{Two simple models with residual stress}

The dependence of material properties on residual stress and, in general, the character of residual stress is not well understood, particularly in the case of soft materials such as elastomers and biological tissues. Some information is obtainable from the opening angle method, but it is qualitative rather than reliably quantitative. For these reasons it is inappropriate to adopt a very general constitutive law that includes all the invariants that depend on the residual stress. Indeed, it suffices to illustrate the effect of residual stress on the solution of boundary-value problems by considering prototype strain-energy functions that depend on the residual stress in the simplest possible way.

We therefore restrict attention to strain-energy functions that consist of a basic neo-Hookean isotropic energy function supplemented by a term linear in either $I_{5}$ or $I_{6}$, and hence linear in $\tau$. Thus, we consider the strain-energy functions defined by

$$
W=\frac{1}{2} \mu\left(I_{1}-3\right)+\frac{1}{2}\left(I_{5}-\operatorname{tr} \tau\right)
$$

and

$$
W=\frac{1}{2} \mu\left(I_{1}-3\right)+\frac{1}{4}\left(I_{6}-\operatorname{tr} \boldsymbol{\tau}\right),
$$

where $\mu(>0)$ is a constant, which corresponds to the shear modulus in the undeformed configuration of a neo-Hookean (isotropic) material. The second term is the strain energy associated with the residual stress and we have taken account of the restriction $(32)_{2}$. The invariants $I_{1}, I_{5}$ and $I_{6}$ are given by (33). Both models were used in [41]. The former is a particular case of a model quadratic in $I_{5}-\operatorname{tr} \tau$ used in [35] and [37], and was also adopted in [42].

The expression (29) for the Cauchy stress simplifies to

$$
\boldsymbol{\sigma}=\mu \mathbf{B}+\boldsymbol{\Sigma}-p \mathbf{I}, \quad \boldsymbol{\sigma}=\mu \mathbf{B}+\frac{1}{2}(\boldsymbol{\Sigma} \mathbf{B}+\mathbf{B} \boldsymbol{\Sigma})-p \mathbf{I},
$$

respectively, for these two energy functions, so that the residual stress is accounted for in somewhat different ways. The components of $\boldsymbol{\sigma}$ can be read off by using the expressions for $\mathbf{B}$ given in (12) and the components of $\boldsymbol{\Sigma}$ given just above Eq. (35), but the separate components are not needed here. Only the expressions derived from (35) are required.

In conjunction with these models it suffices to adopt a specific form of the residual stress component $\tau_{R R}$ that satisfies the boundary conditions (18). We therefore choose the simple form

$$
\tau_{R R}=\alpha(R-A)(R-B),
$$

and hence, from $(17), \tau_{\Theta \Theta}$ is given by

$$
\tau_{\Theta \Theta}=\alpha\left[3 R^{2}-2(A+B) R+A B\right],
$$

where $\alpha$ is a constant which defines the strength of the residual stress. Note that $\tau_{R R}<0(>0)$ if $\alpha>0(<0)$ and that the mean value of $\tau_{\Theta \Theta}$ through the thickness of the tube vanishes.

In Fig. 1 representative plots of $\bar{\tau}_{R R}=\tau_{R R} / \alpha$ and $\bar{\tau}_{\Theta \Theta}=\tau_{\Theta \Theta} / \alpha$ are shown as functions of $R^{*}=R / A$ for four different values of $\eta=B / A$. We observe, in particular, that for $\eta=1.2$ in Fig. 1(a), a value appropriate for arterial walls, the plots are very similar to those arising from the opening angle method (see, for example, [27]). Typical values of $\eta$ for the examples of arteries 


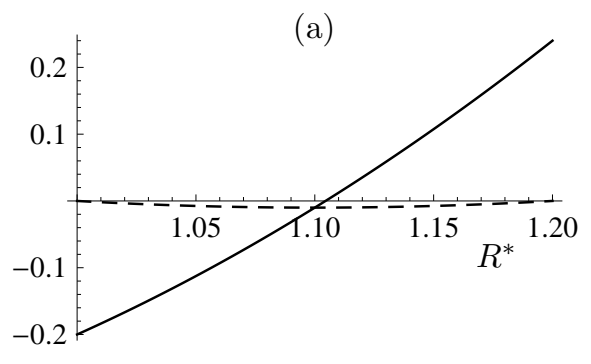

(c)

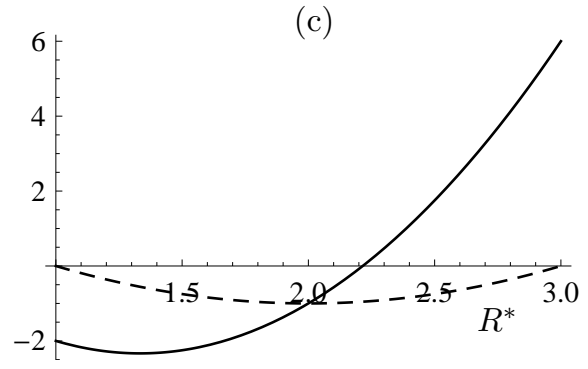

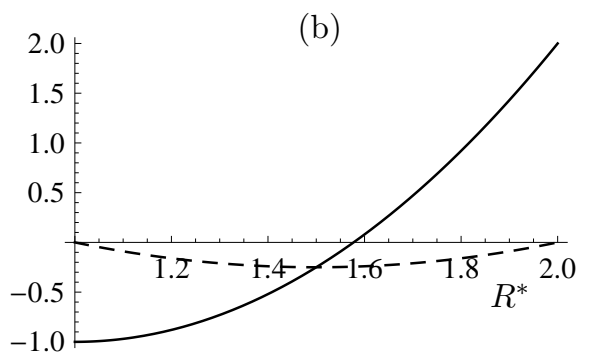

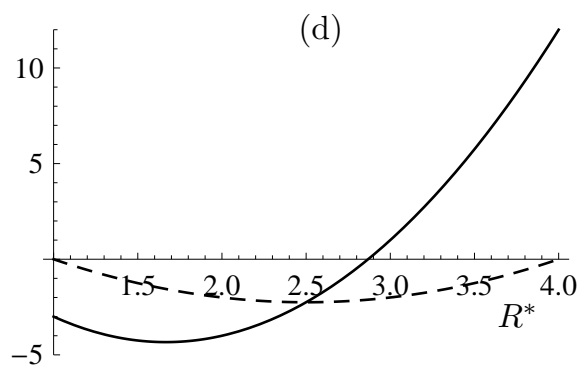

Figure 1: Plots of $\bar{\tau}_{R R}=\tau_{R R} / \alpha$ (dashed curves) and $\bar{\tau}_{\Theta \Theta}=\tau_{\Theta \Theta} / \alpha$ (continuous curves) versus $R^{*}=R / A$ for different values of $\eta=B / A$ : (a) 1.2 ; (b) 2 ; (c) 3 ; (d) 4

considered in Holzapfel et al. [25] are in the range 1.2-1.5. Note the changes in the magnitude and the character of $\bar{\tau}_{\Theta \Theta}$ as the wall thickness increases. In particular, it develops a minimum.

Bearing in mind the expressions (38), (39) and (42) for $P, M$ and $F$, respectively, we obtain for $(43)$

$$
\begin{aligned}
\lambda_{\theta} \hat{W}_{\lambda_{\theta}} & =\mu\left(\lambda_{\theta}^{2}-\lambda_{\theta}^{-2} \lambda_{z}^{-2}\right)+\lambda_{\theta}^{2} \tau_{\Theta \Theta}-\lambda_{\theta}^{-2} \lambda_{z}^{-2} \tau_{R R} \\
\lambda_{z} \hat{W}_{\lambda_{z}} & =\mu\left[\left(1+\gamma^{2}\right) \lambda_{z}^{2}-\lambda_{\theta}^{-2} \lambda_{z}^{-2}\right]-\lambda_{\theta}^{-2} \lambda_{z}^{-2} \tau_{R R} \\
\hat{W}_{\gamma} & =\mu \gamma \lambda_{z}^{2}
\end{aligned}
$$

and for (44)

$$
\begin{aligned}
\lambda_{\theta} \hat{W}_{\lambda_{\theta}} & =\mu\left(\lambda_{\theta}^{2}-\lambda_{\theta}^{-2} \lambda_{z}^{-2}\right)+\left(\lambda_{\theta}^{4}+\frac{1}{2} \gamma^{2} \lambda_{\theta}^{2} \lambda_{z}^{2}\right) \tau_{\Theta \Theta}-\lambda_{\theta}^{-4} \lambda_{z}^{-4} \tau_{R R} \\
\lambda_{z} \hat{W}_{\lambda_{z}} & =\mu\left[\left(1+\gamma^{2}\right) \lambda_{z}^{2}-\lambda_{\theta}^{-2} \lambda_{z}^{-2}\right]+\frac{1}{2} \gamma^{2} \lambda_{\theta}^{2} \lambda_{z}^{2} \tau_{\Theta \Theta}-\lambda_{\theta}^{-4} \lambda_{z}^{-4} \tau_{R R} \\
\hat{W}_{\gamma} & =\mu \gamma \lambda_{z}^{2}+\frac{1}{2} \gamma \lambda_{\theta}^{2} \lambda_{z}^{2} \tau_{\Theta \Theta}
\end{aligned}
$$

For each of the two models we give results for $P, M$ and $F$ in the dimensionless forms $P^{*}, M^{*}$ and $F^{*}$ defined by

$$
P^{*}=\frac{P}{\mu}, \quad M^{*}=\frac{M}{\pi \mu A^{3}}, \quad F^{*}=\frac{F}{\pi \mu A^{2}},
$$

and we also define the dimensionless quantities

$$
\eta=B / A, \quad \psi^{*}=\psi A, \quad \alpha^{*}=\alpha A^{2} / \mu, \quad \lambda_{a}=a / A, \quad e=\lambda_{a}^{2} \lambda_{z}-1 .
$$

It is convenient to write

$$
P^{*}=P_{0}^{*}+P_{i}^{*}, \quad M^{*}=M_{0}^{*}+M_{i}^{*}, \quad F^{*}=F_{0}^{*}+F_{i}^{*},
$$

where $i=5$ and 6 for (43) and (44), respectively, the terms with the zero subscript corresponding to the results without residual stress. 
The integrals in (38), (39) and (42) can all be evaluated explicitly and give (after some detailed but elementary calculations)

$$
\begin{aligned}
P_{0}^{*} & =-\frac{1}{2} \lambda_{z}^{-1} \log \left[\frac{\eta^{2}+e}{\eta^{2}(1+e)}\right]+\frac{1}{2} \lambda_{z}^{-1} \frac{\left(\eta^{2}-1\right) e}{\left(\eta^{2}+e\right)(1+e)}+\frac{1}{2} \lambda_{z} \psi^{* 2}\left(\eta^{2}-1\right), \\
P_{5}^{*} & =-\frac{1}{2} \lambda_{z}^{-1} \alpha^{*} \eta \log \left[\frac{\eta^{2}+e}{\eta^{2}(1+e)}\right]+\alpha^{*} \lambda_{z}^{-1} e \log \left[\frac{\eta^{2}+e}{1+e}\right] \\
& -\frac{3}{2} \alpha^{*} \lambda_{z}^{-1}(\eta+1) e^{1 / 2} \tan ^{-1}\left[\frac{(\eta-1) e^{1 / 2}}{\eta+e}\right], \\
P_{6}^{*}= & \alpha^{*} \lambda_{z}^{-2}\left[\eta+3 e+\lambda_{z}^{2} \psi^{* 2} \eta e\right] \log \eta-\frac{3}{2} \alpha^{*} \lambda_{z}^{-2} \frac{\left(\eta^{2}-1\right) e}{\eta} \\
+ & \frac{1}{12} \alpha^{*} \psi^{* 2}\left(\eta^{2}-1\right)\left[(\eta-1)^{2}-6 e\right]-\frac{1}{2} \alpha^{*} \lambda_{z}^{-2}(\eta-3 e) \log \left[\frac{\eta^{2}+e}{1+e}\right] \\
& -\frac{1}{8} \alpha^{*} \lambda_{z}^{-2} \frac{\left(\eta^{2}-1\right)(\eta+e) e}{\left(\eta^{2}+e\right)(1+e)}-\frac{15}{8} \alpha^{*} \lambda_{z}^{-2}(\eta+1) e^{1 / 2} \tan ^{-1}\left[\frac{(\eta-1) e^{1 / 2}}{\eta+e}\right], \\
M_{0}^{*} & =\frac{1}{2} \psi^{*}\left(\eta^{2}-1\right)\left(2 e+\eta^{2}+1\right), \quad M_{5}^{*}=0, \\
M_{6}^{*} & =\frac{1}{60} \psi^{*} \alpha^{*} \lambda_{z}^{-1}\left(\eta^{2}-1\right)\left[3(\eta-1)^{2}\left(2 \eta^{2}+\eta+2\right)+10(\eta-1)^{2} e-30 e^{2}\right] \\
& +\psi^{*} \alpha^{*} \lambda_{z}^{-1} \eta e^{2} \log \eta,
\end{aligned}
$$

and

$$
\begin{aligned}
F_{0}^{*} & =-\frac{1}{2} \psi^{*} M_{0}^{*}+\left(\eta^{2}-1\right)\left(\lambda_{z}-\lambda_{z}^{-2}\right)+\frac{1}{2} \lambda_{z}^{-2} e \log \left[\frac{\eta^{2}+e}{\eta^{2}(1+e)}\right] \\
F_{5}^{*} & =\frac{1}{2} \alpha^{*} \lambda_{z}^{-2} \eta e \log \left[\frac{\eta^{2}+e}{\eta^{2}(1+e)}\right]-\frac{1}{2} \alpha^{*} \lambda_{z}^{-2} e^{2} \log \left[\frac{\eta^{2}+e}{1+e}\right] \\
& +\alpha^{*} \lambda_{z}^{-2}(\eta+1) e^{3 / 2} \tan ^{-1}\left[\frac{(\eta-1) e^{1 / 2}}{\eta+e}\right] \\
F_{6}^{*} & =\frac{3}{2} \alpha^{*} \lambda_{z}^{-3} \frac{\left(\eta^{2}-1\right) e^{2}}{\eta}-\frac{3}{2} \alpha^{*} \lambda_{z}^{-3} e^{2} \log \left[\frac{\eta^{2}\left(\eta^{2}+e\right)}{1+e}\right]-\psi^{*} M_{6}^{*} \\
& +\alpha^{*} \lambda_{z}^{-3} \eta e \log \left[\frac{\eta^{2}+e}{\eta^{2}(1+e)}\right]+\frac{5}{2} \alpha^{*} \lambda_{z}^{-3}(\eta+1) e^{3 / 2} \tan ^{-1}\left[\frac{(\eta-1) e^{1 / 2}}{\eta+e}\right] .
\end{aligned}
$$

For a solid cylinder, obtained by taking the limit $A \rightarrow 0$ (and $a \rightarrow 0$ ), the deformation (8) 1 reduces to $r=\lambda_{z}^{-1 / 2} R$ and hence $\lambda_{r}=\lambda_{\theta}=\lambda_{z}^{-1 / 2}$ (the deformation corresponds to that associated with simple tension), and hence $e=0$, we obtain for the model (43) the classical results for a neo-Hookean material (see [15], in which results were given for the Mooney-Rivlin material):

$$
M_{0}=\frac{1}{2} \pi \mu \psi B^{4}, \quad F_{0}=\pi \mu\left(\lambda_{z}-\lambda_{z}^{-2}\right) B^{2}-\frac{1}{2} \psi M_{0} .
$$

The residual stress does not affect these results $\left(M_{5}=F_{5}=0\right)$.

On the other hand, for the model (44), we obtain for a solid cylinder $M=M_{0}+M_{6}, F=F_{0}+F_{6}$, where

$$
M_{6}=\frac{1}{10} \pi \alpha \psi \lambda_{z}^{-1} B^{6}, \quad F_{6}=-\psi M_{6} .
$$

Thus, the residual stress does have an influence in this case. The latter results agree with those obtained in [41] for a solid cylinder. The notation $A$ was used instead of $B$ and $\lambda$ instead of $\lambda_{z}$ 

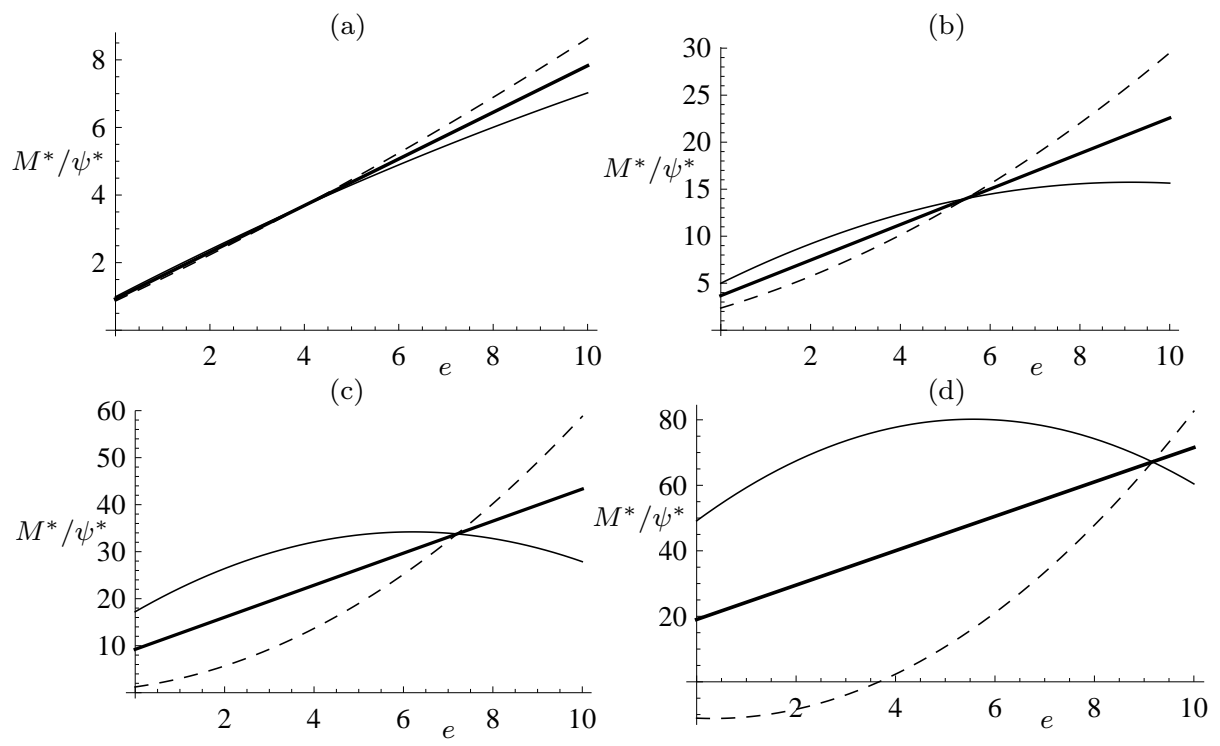

Figure 2: Plots of $M^{*} / \psi^{*}$ versus $e$ for $\lambda_{z}=1$ and $\alpha^{*}=3,0,-3$ (continuous, thick continuous and dashed curves, respectively) with the following values of $\eta$ : (a) 1.3 ; (b) 1.7 ; (c) 2.1 ; (d) 2.5 .

in equations (82) and (84) of [41], and the $\alpha$ therein has the opposite sign to that used here. Moreover, $\lambda^{-2}$ instead of $\lambda^{-1}$ appeared incorrectly in the expressions involving $A^{6}$ in [41].

In the following we provide numerical results to illustrate the dependence of $P^{*}, M^{*}$ and $F^{*}$ on $e$, which is a measure of the radial expansion of the tube for a given axial stretch $\lambda_{z}$, for various fixed values of $\eta, \lambda_{z}, \psi^{*}$ and $\alpha^{*}$.

\subsection{Numerical results}

First, since $M^{*}$ is proportional to $\psi^{*}$, we plot $M^{*} / \psi^{*}$ versus $e$ for $M^{*}=M_{0}^{*}+M_{6}^{*}$ based on Eq. (54), noting that $M_{5}^{*}=0$. In Fig. 2, $M^{*} / \psi^{*}$ is shown as a function of $e$ for $\lambda_{z}=1$, for four values of $\eta$ with $\alpha^{*}=3,0,-3$ in each case, while in Fig. $3 M^{*} / \psi^{*}$ is shown as a function of $e$ for $\lambda_{z}=1$, for $\eta=1.7$ and for four separate sets of values of $\alpha^{*}$. Note that $e=\lambda_{a}^{2} \lambda_{z}-1$ captures dependence on the radius, through $\lambda_{a}$, and, in general, the axial stretch $\lambda_{z}$, and that results in the absence of residual stress correspond to $\alpha^{*}=0$. In the latter case $M^{*} / \psi^{*}$ is linear in $e$, as is clear from the thick continuous lines in Figs. 2 and 3, but depends strongly on the tube thickness via $\eta$.

In Fig. 2 we see that $M^{*} / \psi^{*}$ increases with the thickness of the tube for positive $\alpha^{*}$, develops a maximum and, for sufficiently large $e$, would become negative, so that $M^{*}$ and $\psi^{*}$ have opposite signs. Thus, according to the model (44), increasing inflation counteracts the effect of torsion and ultimately reverses it. On the other hand, for negative $\alpha^{*}, M^{*} / \psi^{*}$ becomes negative for small $e$ when the tube thickness is sufficiently large. A similar trend can be seen in Fig. 3 with increasing magnitude of $\alpha^{*}$ at a fixed value of the tube thickness. These results suggest, intuitively, that the predictions of the model (44) run counter to physical expectations for residual stresses beyond a certain magnitude. This is consistent with the requirements of strong ellipticity considered in Sect. 7, which impose restrictions on the possible range of values of $\alpha^{*}$.

Results for $M^{*}$ itself can, of course, be read off from these graphs for any given value of $\psi^{*}$. Note that the differences between the results for the different values of $\alpha^{*}$ in Fig. 2 increase with the thickness of the tube, while for a fixed tube thickness in Fig. 3 the differences in the curves for the three values of $\alpha^{*}$ in each panel increase with the magnitude of $\alpha^{*}$, and a maximum develops as positive $\alpha^{*}$ increases. Thus, in summary, the effect of the residual stress is greater for thick than for thin tubes, and, not surprisingly, the material response in torsion is more affected by a large residual stress than by a smaller one. 

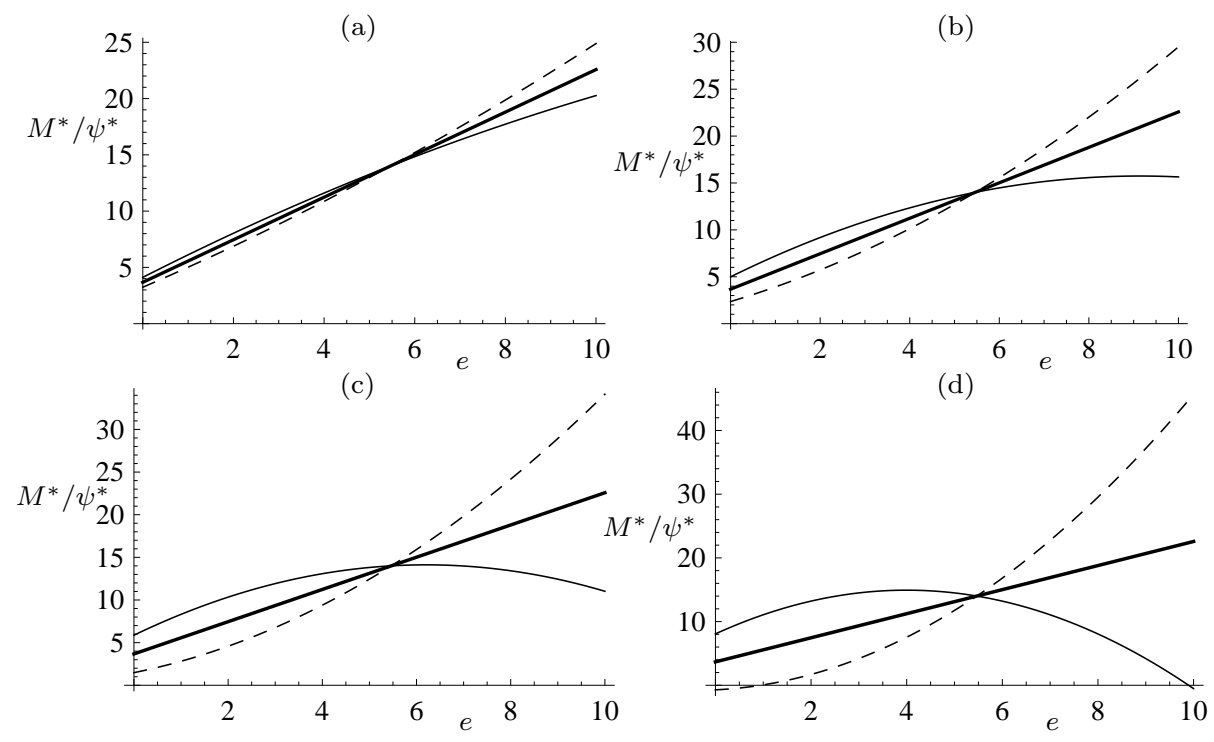

Figure 3: Plots of $M^{*} / \psi^{*}$ versus $e$ for $\lambda_{z}=1$ and $\eta=1.7$ with the following values of $\alpha^{*}$ : (a) $1,0,-1$; (b) $3,0,-3$; (c) $5,0,-5$; (d) $10,0,-10$, corresponding in each panel to the continuous, thick continuous and dashed curves, respectively.

Note that $\lambda_{z}$ appears in $M_{6}^{*}$ only as a factor $\lambda_{z}^{-1}$ and therefore does not have a significant influence on the behaviour of $M_{6}^{*}$. On the other hand, $\lambda_{z}$ does not appear explicitly in $M_{0}^{*}$. Nevertheless, as our calculations have shown for a series of values of $\lambda_{z}$, the inclusion of $\lambda_{z} \neq 1$ in $M^{*}$ does not affect the qualitative features of Figs. 2 and 3, and we do not therefore include separate plots of $M^{*}$ for $\lambda_{z} \neq 1$. A change in $\lambda_{z}$ has only a minor numerical influence on $M^{*}$, and the results for $\lambda_{z}=1$ are intermediate between those for $\lambda_{z}>1$ and $\lambda_{z}<1$.

We have found that this is also the case for $P^{*}$ and $F^{*}$, and we therefore illustrate the results for $P^{*}$ and $F^{*}$ by setting $\lambda_{z}=1$.

We recall that $M_{5}^{*}$ does not depend on the residual stress, while $M_{6}^{*}$ does. By contrast, $P_{5}^{*}$ and $F_{5}^{*}$ do depend on the residual stress, as do $P_{6}^{*}$ and $F_{6}^{*}$. In the following, therefore, we illustrate the behaviour of both $P^{*}$ and $F^{*}$ in each case. First, in Fig. 4, for the representative value $\eta=1.5$ of the tube thickness ratio, $P^{*}=P_{0}^{*}+P_{5}^{*}$ is plotted against $e$ for four different values of the torsional strain $\psi^{*}$, with $\alpha^{*}=-5,0,5$ in each case. The plots in Fig. 4(a) correspond to pure inflation $\left(\psi^{*}=0\right)$. For a non-zero value of $\psi^{*}$ the curves start at $e<0$ because, when a torsion is applied at zero pressure $e$ decreases, i.e. the (inner) radius of the tube decreases. Without residual stress $\left(\alpha^{*}=0\right)$ the curves correspond to the well-known neo-Hookean behaviour. When residual stress is present the behaviour is qualitatively similar, a positive (negative) $\alpha^{*}$ reducing (increasing) the pressure required to achieve a given inflation but the pressure increases to a finite asymptotic value in each case. The difference in the results for $\alpha^{*}=0$ and $\alpha^{*} \neq 0$ is most pronounced for small $\psi^{*}$ and decreases as $\psi^{*}$ increases. Increasing torsion causes the pressure response to be stiffer, i.e. for a larger $\psi^{*}$ a larger $P^{*}$ is required to produce a given value of $e$.

Next, in Fig. 5, we plot $P^{*}=P_{0}^{*}+P_{6}^{*}$ against $e$ for the same $\eta$ and $\psi^{*}$ but a smaller $\alpha^{*}$. For a negative value of $\alpha^{*}$, the pressure increases monotonically with $e$, so the residual stress in this case might be considered to have a stabilizing influence. On the other hand, a positive $\alpha^{*}$ causes the pressure to have a maximum, which then reduces to zero and becomes negative. This is physically unrealistic, and therefore suggests that the model (44) is limited to relatively small values of $e$ when $\alpha^{*}>0$. As with Fig. 4, for a larger $\psi^{*}$ a larger $P^{*}$ is required to produce a given value of $e$.

We now illustrate the behaviour of the reduced axial load in the dimensionless form $F^{*}$, noting that $F^{*}$ is required to maintain the axial length of the tube with $\lambda_{z}=1$ when it is subject to 

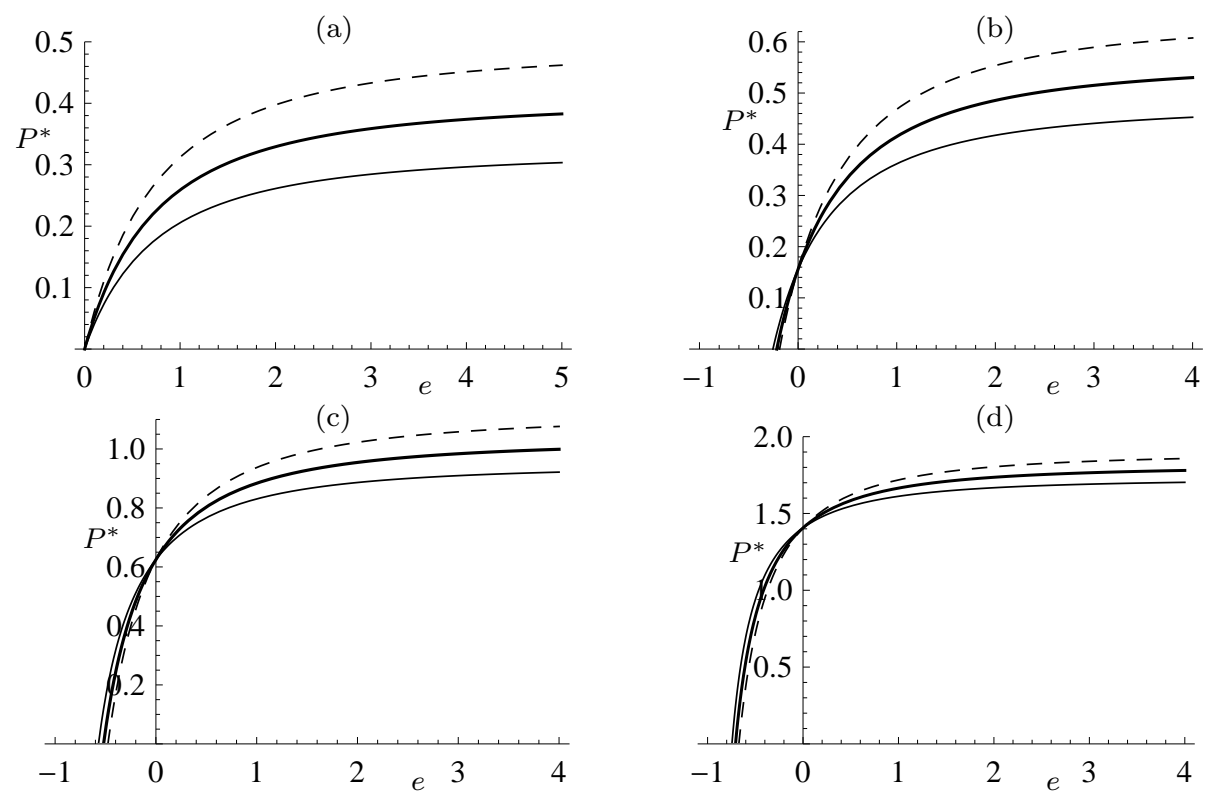

Figure 4: Plots of $P^{*}=P_{0}^{*}+P_{5}^{*}$ versus $e$ for $\lambda_{z}=1$ and $\eta=1.5$, with the following values of $\psi^{*}$ : (a) 0 ; (b) 0.5 ; (c) 1 ; (d) 1.5. In each case the continuous, thick continuous and dashed curves correspond to $\alpha^{*}=5,0,-5$.
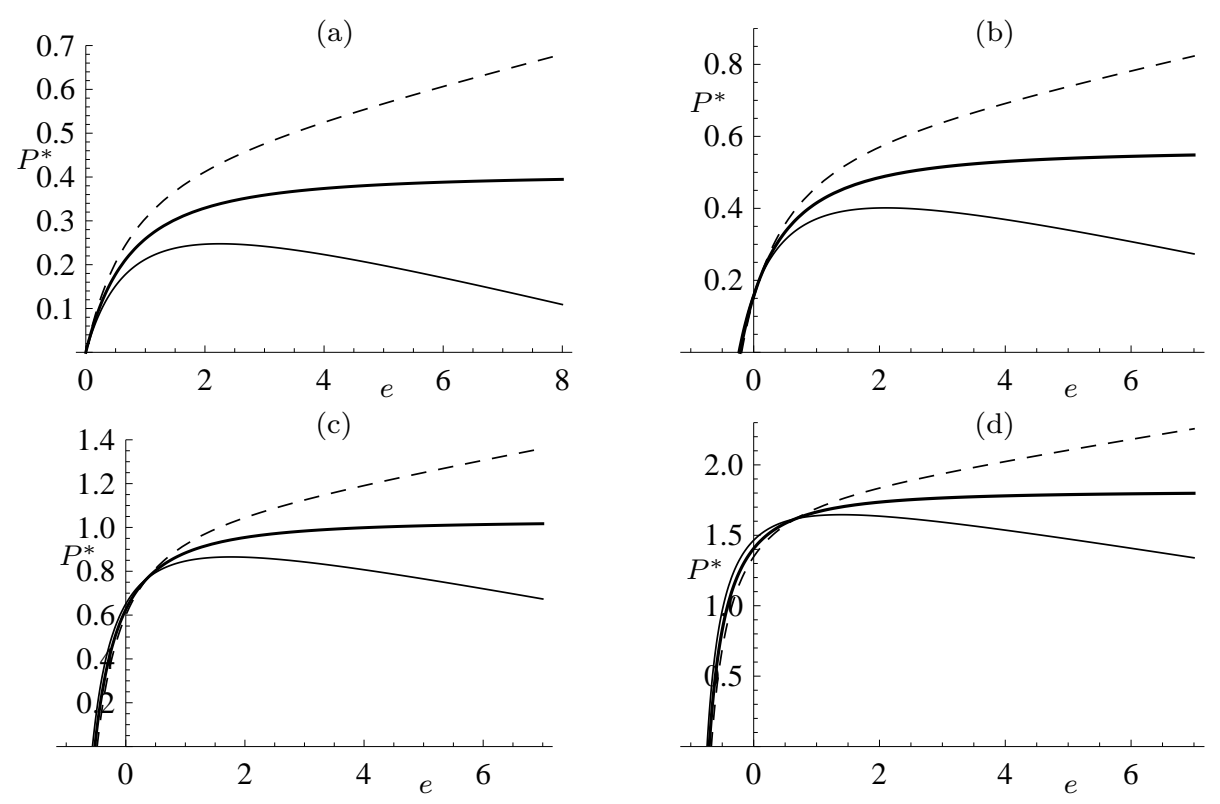

Figure 5: Plots of $P^{*}=P_{0}^{*}+P_{6}^{*}$ versus $e$ for $\lambda_{z}=1$ and $\eta=1.5$, with the following values of $\psi^{*}$ : (a) 0 ; (b) 0.5 ; (c) 1 ; (d) 1.5. In each case the continuous, thick continuous and dashed curves correspond to $\alpha^{*}=1,0,-1$.

torsion and internal pressure. In Fig. $6, F^{*}=F_{0}^{*}+F_{5}^{*}$ is plotted against $e$. The curves start on the left at a value of $e$ corresponding to zero pressure, and the four panels correspond to different values of the applied torsional strain $\psi^{*}$. As for $P^{*}$, the residual stress has a significant effect for small torsion but less so as the magnitude of the torsion increases. In each case $F^{*}$ is negative so the tube would extend under the combined pressure and torsion if it were free to do so without 
(a)

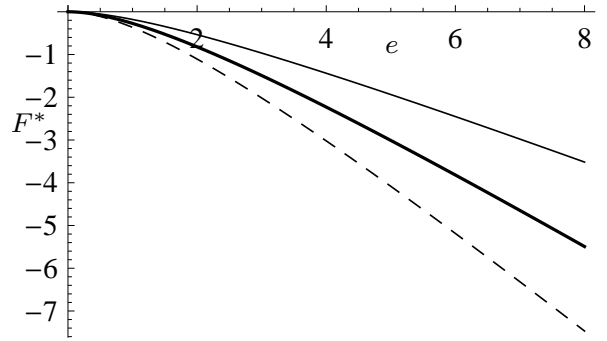

(c)

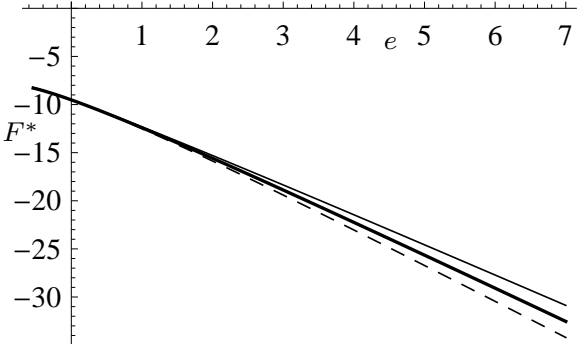

(b)

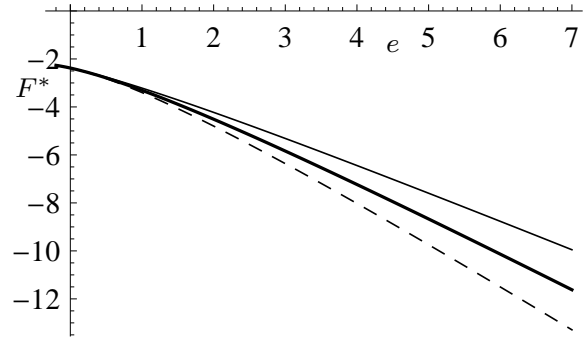

(d)

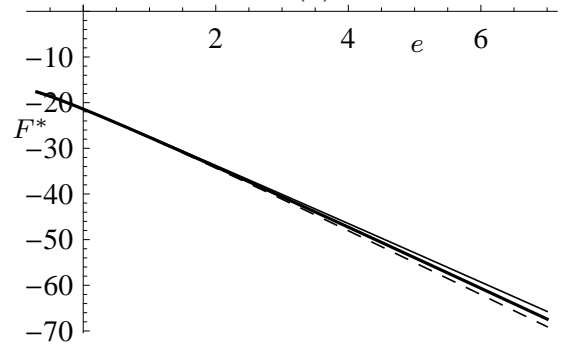

Figure 6: Plots of $F^{*}=F_{0}^{*}+F_{5}^{*}$ versus $e$ for $\lambda_{z}=1$ and $\eta=2.5$ with the following values of $\psi^{*}$ : (a) 0 ; (b) 0.5 ; (c) 1 ; (d) 1.5. In each case the continuous, thick continuous and dashed curves correspond to $\alpha^{*}=1,0,-1$.

the application of $F^{*}$, a negative value of which is required to prevent an increase in $\lambda_{z}$. The magnitude $\left|F^{*}\right|$ increases with the magnitude of the torsion $\psi^{*}$.

The behaviour shown in Fig. 6 has some similarities with that for a fibre-reinforced tube under inflation and torsion. For example, for a neo-Hookean material supplemented by the standard reinforcing model and radial reinforcement, $F^{*}$ becomes negative as $e$ increases, although it is positive as inflation begins and then reaches a maximum before monotonically decreasing; see, for example, El Hamdaoui et al. [20] for discussion of the present problem for fibre-reinforced materials.

Plots of $F^{*}=F_{0}^{*}+F_{6}^{*}$ are shown in Fig. 7 for the same values of all the parameters as in Fig. 6. The behaviour shown here is generally quite different from that shown in Fig. 6 when residual stress is present. For $\psi^{*}=0$, for example, a positive $\alpha^{*}$ leads to a positive $F^{*}$, even for small values of $e$ (for which the $P^{*}$ results are realistic), while for negative $\alpha^{*}$ the behaviour of $F^{*}$ is qualitatively similar to that shown in Fig. 6, although numerically somewhat different. This changes significantly as $\psi^{*}$ increases, when, for positive $\alpha^{*}, F^{*}$ is negative as inflation begins but then deceases to a minimum as $e$ increases and ultimately becomes positive. On the other hand, for negative $\alpha^{*}, F^{*}$ is slightly positive for small $e$, reaches a maximum and then quickly becomes negative and monotonically decreasing as $e$ increases. The qualitative features in Fig. 7 remain as the magnitude of $\alpha^{*}$ increases, but, interestingly, our calculations show that for larger positive $\alpha^{*}, F^{*}$ in Fig. 6(a) is positive and monotonically increasing with $e$, as in Fig. 7(a).

\section{Concluding remarks}

It should be emphasised that the results highlighted in the previous section are for very simple models of elasticity incorporating residual stress. These simple models may not be truly representative of the effect of residual stress, but, at present, there are not adequate quantitative data available to inform or justify the development of more elaborate models.

It is clear from the illustrations that the considered residual stress can have a significant effect on the elastic response of a tube, in some cases improving performance but in other examples weakening the material response, possibly destabilising the material and leading to counterintuitive 

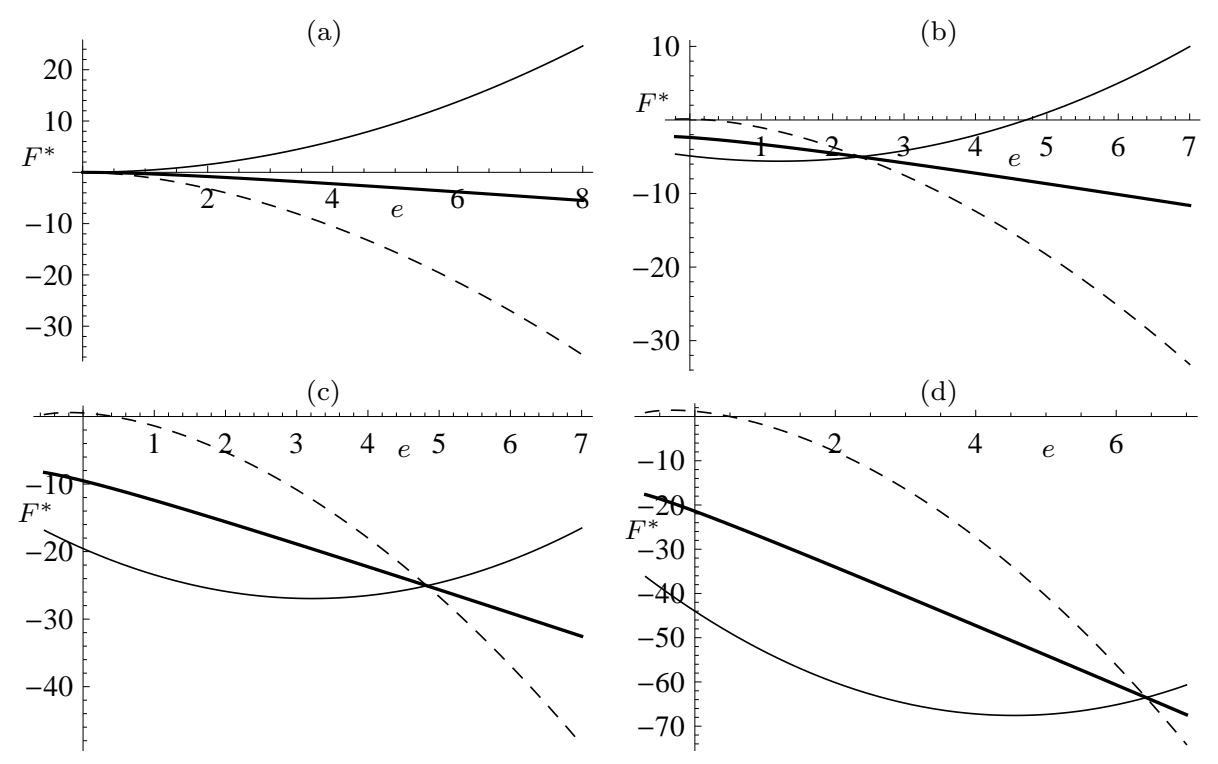

Figure 7: Plots of $F^{*}=F_{0}^{*}+F_{6}^{*}$ versus $e$ for $\lambda_{z}=1$ and $\eta=2.5$ with the following values of $\psi^{*}$ : (a) 0 ; (b) 0.5 ; (c) 1 ; (d) 1.5. In each case the continuous, thick continuous and dashed curves correspond to $\alpha^{*}=1,0,-1$.

and what might be considered unphysical behaviour. A thorough analysis of the influence of residual stress on stability is needed, but is beyond the scope of the present work.

We do, however, provide a brief analysis of strong ellipticity here since it is a local concept that can be applied to the models (43) and (44) themselves without reference to the geometry of the considered problem. For an incompressible material the strong ellipticity condition has the form

$$
[\mathbf{Q}(\mathbf{n}) \mathbf{m}] \cdot \mathbf{m}>0 \quad \text { for all non-zero vectors } \mathbf{m} \text { and } \mathbf{n} \text { such that } \mathbf{m} \cdot \mathbf{n}=0,
$$

where $\mathbf{Q}(\mathbf{n})$ is the acoustic tensor, which is given, in Cartesian components, by $Q_{i j}(\mathbf{n})=\mathcal{A}_{p i q j} n_{p} n_{q}$ (with summation over indices $p$ and $q$ from 1 to 3 ), $\mathcal{A}_{p i q j}$ being the components of the elasticity tensor. For a residually stressed material the latter components were given in [35], and in general, the component expressions are very lengthy and therefore not reproduced here. We focus on the specializations of the strong ellipticity condition for the models (43) and (44). First, for (43), the strong ellipticity condition obtained by using the expression for $\mathcal{A}_{p i q j}$ in [35] is independent of $\mathbf{m}$ and has the form

$$
\mu(\mathbf{B n}) \cdot \mathbf{n}+(\mathbf{\Sigma} \mathbf{n}) \cdot \mathbf{n}>0, \quad \mathbf{n} \neq \mathbf{0} .
$$

For the considered deformation and residual stress, this specializes to

$$
\left(\mu+\tau_{R R}\right) \lambda_{r}^{2} n_{r}^{2}+\left(\mu+\tau_{\Theta \Theta}\right) \lambda_{\theta}^{2} n_{\theta}^{2}+\mu \lambda_{z}^{2}\left(\gamma n_{\theta}+n_{z}\right)^{2}>0, \quad\left(n_{r}, n_{\theta}, n_{z}\right) \neq(0,0,0),
$$

where $\left(n_{r}, n_{\theta}, n_{z}\right)$ are the components of $\mathbf{n}$. Necessary and sufficient conditions for (60) to hold are simply

$$
\mu+\tau_{R R}>0, \quad \mu+\tau_{\Theta \Theta}>0,
$$

independently of the deformation.

For the specific forms of $\tau_{R R}$ and $\tau_{\Theta \Theta}$ given in (46) and (47) with $R \in[A, B]$, the strong ellipticity inequalities

$$
-\frac{1}{\eta(\eta-1)}<\alpha^{*}<\frac{3}{1-\eta+\eta^{2}}
$$

may be deduced, where we recall that $\eta=B / A$ and $\alpha^{*}=\alpha A^{2} / \mu$. For $\eta=1.5$ we obtain $-4 / 3<\alpha^{*}<12 / 7$, which corrects the inequalities (59) given in [41]. Note that the $\alpha^{*}$ here 
corresponds to the notation $-\bar{\alpha}$ used in [41]. For a solid cylinder $(A \rightarrow 0)$ the inequalities $(62)$ reduce to $-\mu<\alpha B^{2}<3 \mu$.

For the model (44), the counterpart of (59), obtained from the formula in [35], is

$$
\begin{aligned}
\mu(\mathbf{B n}) \cdot \mathbf{n} & +(\mathbf{\Sigma} \mathbf{B n}) \cdot \mathbf{n}+(\mathbf{\Sigma m}) \cdot \mathbf{n}(\mathbf{B m}) \cdot \mathbf{n} \\
& +\frac{1}{2}[(\mathbf{\Sigma} \mathbf{n}) \cdot \mathbf{n}(\mathbf{B m}) \cdot \mathbf{m}+(\mathbf{\Sigma} \mathbf{m}) \cdot \mathbf{m}(\mathbf{B n}) \cdot \mathbf{n}]>0,
\end{aligned}
$$

with $\mathbf{m} \cdot \mathbf{n}=0, \mathbf{m} \neq \mathbf{0}, \mathbf{n} \neq \mathbf{0}$. This depends in a relatively complicated way on the deformation, and it is not possible to extract simple necessary and sufficient conditions in general in this case. When evaluated in the reference configuration, however, necessary and sufficient conditions are found to be

$$
\mu+\frac{1}{2}\left(3 \tau_{R R}+\tau_{\Theta \Theta}\right)>0, \quad \mu+\frac{1}{2}\left(\tau_{R R}+3 \tau_{\Theta \Theta}\right)>0 .
$$

Again using the specific forms of $\tau_{R R}$ and $\tau_{\Theta \Theta}$ given in (46) and (47), restrictions on the parameters $\alpha^{*}$ and $\eta$ that guarantee strong ellipticity are obtained as

$$
-\frac{2}{3} \frac{1}{\eta(\eta-1)}<\alpha^{*}<\frac{80}{49(\eta+1)^{2}-160 \eta}
$$

which are more restrictive than (62). For a solid cylinder these become $-2 \mu / 3<\alpha B^{2}<80 \mu / 49$.

Some values of $\alpha^{*}$ used for illustration in the previous section are outside the ranges of values appropriate for satisfaction of the strong ellipticity inequalities above, but for values within these ranges the behaviour is similar qualitatively.

\section{Acknowledgements}

The authors acknowledge support from the Ministerio de Ciencia, Spain, under the project number DPI2011-26167.

\section{References}

[1] Rivlin, R.S.: 1947. Torsion of a rubber cylinder. J. Appl. Phys. 18, 444-449 (1947)

[2] Rivlin, R.S.: 1948. Large elastic deformations of isotropic materials IV. Further developments of the general theory. Phil. Trans. R. Soc. Lond. A 241, 379-397 (1948)

[3] Rivlin, R.S.: A note on the torsion of an incompressible highly-elastic cylinder. Math. Proc. Cambridge Phil. Soc. 45, 485-487 (1949)

[4] Rivlin, R.S., Saunders, D.W.: Large elastic deformations of isotropic materials VII. Experiments on the deformation of rubber. Phil. Trans. R. Soc. Lond. A 243, 251-288 (1951)

[5] Gent, A.N., Rivlin, R.S.: Experiments on the mechanics of rubber II: The torsion, inflation and extension of a tube. Proc. Phys. Soc. B 65, 487-501 (1952)

[6] Green, A.E., Adkins, J.E.: Large elastic deformations, second edition, Clarendon Press, Oxford (1970)

[7] Ogden, R.W., Chadwick, P.: On the deformation of solid and tubular cylinders of incompressible isotropic elastic materials. J. Mech. Phys. Solids. 20, 77-90 (1972)

[8] Horgan, C.O., Polignone, D.A.: A note on the pure torsion of a circular cylinder for a compressible nonlinearly elastic material with nonconvex strain-energy. J. Elasticity 37, 167$178(1995)$

[9] Kirkinis, E., Ogden, R.W.: On extension and torsion of a compressible elastic circular cylinder. Math. Mech. Solids 7, 373-392 (2002) 
[10] Polignone, D.A., Horgan, C.O.: Pure torsion of compressible non-linearly elastic circular cylinders. Q. Appl. Math. 49, 591-607 (1991)

[11] Horgan, C.O., Saccomandi, G.: Simple torsion of isotropic, hyperelastic, incompressible materials with limiting chain extensibility. J. Elasticity 56, 159-170 (1999)

[12] Kanner, L., Horgan, C.O.: On extension and torsion of strain-stiffening rubber-like elastic circular cylinders. J. Elasticity 93, 39-61 (2008)

[13] Horgan, C.O., Murphy, J.G.: Torsion of incompressible fibre-reinforced nonlinearly elastic circular cylinders. J. Elasticity 103, 235-246 (2011)

[14] Horgan, C.O., Murphy, J.G.: Finite extension and torsion of fiber-reinforced non-linearly elastic circular cylinders. Int. J. Non-Lin. Mech. 47, 97-104 (2012)

[15] Rivlin, R.S.: Large elastic deformations of isotropic materials VI. further results in the theory of torsion, shear and flexure. Phil. Trans. R. Soc. Lond. A 242, 173-195 (1949)

[16] Zidi, M.: Torsion and telescopic shearing of a compressible hyperelastic tube. Mech. Res. Comm. 26, 245-252 (1999)

[17] Zidi, M.: Finite torsion and anti-plane shear of a compressible hyperelastic and transversely isotropic tube. Int. J. Eng. Sci. 38, 1487-1496 (2000)

[18] Zidi, M.: Finite torsion and shearing of a compressible and anisotropic tube. Int. J. Non-Lin. Mech. 35, 1115-1126 (2000)

[19] Zidi, M., Cheref, M.: Finite deformations of fibre-reinforced vascular prosthesis. Mech. Res. Comm. 28, 55-62 (2001)

[20] El Hamdaoui, M., Merodio, J., Ogden, R.W., Rodríguez, J.: Finite elastic deformations of transversely isotropic circular cylindrical tubes. Int. J. Solids and Struct. 51, 1188-1196 (2014)

[21] Paige, R.E.: FEA in the design process of rubber bushings. ABAQUS Users' Confereence, pp. 1-15. Simulia, Dassault Systèmes, Providence, RI (2002)

[22] Paige, R.E., Mars, W.V.: Implications of the Mullins effect on the stiffness of a pre-loaded rubber component. ABAQUS Users' Confereence, pp. 1-15. Simulia, Dassault Systèmes, Providence, RI (2004)

[23] Rachev, A.: Theoretical study of the effect of stress-dependent remodeling on arterial geometry under hypertensive conditions. J. Biomech. 30, 819-827 (1997)

[24] Rachev, A., Hayashi, K.: (1999) Theoretical study of the effects of vascular smooth muscle contraction on strain and stress distributions in arteries. Ann. Biomed. Eng. 27, 459-468 (1999)

[25] Holzapfel, G.A., Gasser, T.C., Ogden, R.W.: A new constitutive framework for arterial wall mechanics and a comparative study of material models. J. Elast. 61, 1-48 (2000)

[26] Ogden, R.W., Schulze-Bauer, C.A.J.: Phenomenological and structural aspects of the mechanical response of arteries. Proceedings of the ASME Mechanics in Biology Symposium, Orlando, November 2000. ASME AMD-Vol. 242/BED-Vol. 46, pp. 125-140. ASME: New York (2000)

[27] Ogden, R.W.: Nonlinear elasticity, anisotropy, material stability and residual stresses in soft tissue (lecture notes, CISM Course on the Biomechanics of Soft Tissue in Cardiovascular Systems), pp. 65-108. CISM Courses and Lectures Series 441, Springer, Wien (2003) 
[28] Holzapfel, G.A., Ogden, R.W.: Modelling the layer-specific 3D residual stresses in arteries, with an application to the human aorta. J. R. Soc. Interface 7, 787-799 (2010)

[29] Zidi, M.: Combined torsion, circular shearing and axial shearing of a compressible hyper elastic and prestressed tube. J. Appl. Mech. 67, 33-40 (2000)

[30] Zidi, M.: Azimuthal shearing and torsion of a compressible and prestressed tube. Int. J. Non-Lin. Mech. 35, 210-209 (2000)

[31] Zidi, M.: Effects of a prestress on a reinforced, nonlinearly elastic and compressible tube subjected to combined deformations. Int. J. Solids Struct. 38, 4657-4669 (2001)

[32] Zidi, M., Cheref, M.: Finite deformations of a hyperelastic, compressible and fibre reinforced tube. Eur. J. Mech. A/Solids 21, 971-980 (2002)

[33] Hoger, A.: On the residual stress possible in an elastic body with material symmetry. Arch. Rational Mech. Anal. 88, 271-290 (1985)

[34] Hoger, A.: The constitutive equation for finite deformations of a transversely isotropic hyperelastic material with residual stress. J. Elast. 33, 107-118 (1993)

[35] Shams, M., Destrade, M., Ogden, R.W.: Initial stresses in elastic solids: constitutive laws and acoustoelasticity. Wave Motion 48, 552-567 (2011)

[36] Ogden, R.W., Singh, B.: Propagation of waves in an incompressible transversely isotropic elastic solid with initial stress: Biot revisited. J. Mech. Mater. Struct. 6, 453-477 (2011)

[37] Shams, M., Ogden, R.W.: On Rayleigh-type surface waves in an initially stressed incompressible elastic solid. IMA J. Appl. Math. 79, 360-376 (2014)

[38] Ogden, R.W., Singh, B.: The effect of rotation and initial stress on the propagation of waves in a transversely isotropic elastic solid. Wave Motion 51, 1108-1126 (2014)

[39] Hoger, A.: On the determination of residual stress in an elastic body. J. Elast. 16, 303-324 (1986)

[40] Johnson, B.E., Hoger, A.: The dependence of the elasticity tensor on residual stress. J. Elast. 33, 145-165 (1993)

[41] Merodio, J., Ogden, R.W., Rodríguez, J.: The influence of residual stress on finite deformation elastic response. Int. J. Non-Lin. Mech. 56, 43-49 (2013)

[42] Ogden, R.W.: Nonlinear elasticity with application to soft fibre-reinforced materials, CISM course on Nonlinear Mechanics of Soft Fibrous Materials. CISM Courses and Lectures Series 559. Springer, Wien (2014)

[43] Ogden, R.W.: Non-linear Elastic Deformations. Dover Publications, New York (1997)

[44] Holzapfel, G.A.: Nonlinear Solid Mechanics. A Continuum Approach for Engineering. John Wiley \& Sons, Chichester (2000)

[45] Spencer, A.J.M.: Theory of invariants, in Continuum Physics Vol. 1, edited by A. C. Eringen, pp. 239-353. Academic Press, New York (1971)

[46] Ogden, R. W. and Singh, B.: Propagation of waves in an incompressible transversely isotropic elastic solid with initial stress: Biot revisited. J. Mech. Mater. Struct. 6, 453-477 (2011) 